\title{
EXPERIMENTAL REINTRODUCTION OF THE THREATENED TERRESTRIAL ORCHID DIURIS FRAGRANTISSIMA
}

\author{
Zoe F. Smith ${ }^{1,3}$, Elizabeth A. James ${ }^{2} \&$ Cassandra B. McLean ${ }^{1}$ \\ ${ }^{1}$ School of Resource Management, Faculty of Land and Food Resources, Burnley College, The University of \\ Melbourne, 500 Yarra Boulevard Richmond, Victoria, 3121 Australia \\ ${ }^{2}$ Royal Botanic Gardens Melbourne, Birdwood Avenue South Yarra, Victoria, 3141 Australia \\ ${ }^{3}$ Author for correspondence: z.smith@pgrad.unimelb.edu.au. Phone 0392506800
}

KEY WORDS: Reintroduction, mycorrhiza, fungal persistence, inoculum, recovery plan

\section{Introduction}

Diuris fragrantissima D. L. Jones et M. A. Clem. is a perennial terrestrial orchid endemic to the state of Victoria, Australia. The species is listed as Critically Endangered in Victoria under the criteria of the International Union for the Conservation of Nature and Natural Resources (IUCN), having suffered a severe population decline since the $1930 \mathrm{~s}$, largely attributable to habitat destruction for agricultural and urban development. A recovery plan has been developed by the Department of Sustainability and the Environment (DSE, Victoria, Australia), which includes reintroduction as an important conservation strategy. There is, however, limited research on terrestrial orchid reintroductions, and many of the previous reintroductions reported have been unsuccessful, including three reintroductions of D. fragrantissima in the $1980 \mathrm{~s}$, in which the majority of plants survived less than 6 months (LaTrobe University, 1982, 1983) (D. Tonkinson pers comm.. 2006). The last of the plants is thought to have died out after 1991 (Murphy et al. 2006), with the exception of an anecdotal report of a flowering plant in 2000 (Knight et al. 2003). The reasons that these trials were unsuccessful are unknown, largely because of a lack of published data. While in situ management should be the first priority for species conservation (Ramsay and Dixon 2003, Vallee et al. 2004), the limited success of management action in the preservation of $D$. fragrantissima at the remnant site, and the continual destruction of known secure habitat, has resulted in reintroduction as a major conservation option. An adaptive management approach involving experimental trials will aid understanding of the system and allow management practices to adapt to the requirements of the orchid.

A comparable level of genetic diversity exists between in situ and ex situ Diuris fragrantissima and its more common relatives (Smith et al. 2006), indicating that the ex situ collection contains sufficient diversity for retaining evolutionary potential to adapt to long-term environmental change, a requirement for the long-term persistence of reintroduced populations (Vallee et al. 2004). In addition, suitable mycorrhizal fungi have been isolated and cultured for use in reintroductions (Smith 2006), which is one of the major steps in the recovery plan (Murphy et al. 2006). The inoculation of habitat soils with suitable mycorrhizal fungi is suggested as a prerequisite to ensure that reintroduced orchids survive the transition from ex situ to in situ (Batty et al. 2001). Furthermore, the reliance of terrestrial orchid seed germination on infection with a mycorrhizal fungus (Batty et al. 2001) means that fungal presence in the soil is required for further in situ seed germination, for development of self-sustaining populations. After reintroduction of orchids and their associated fungi, the persistence of the mycorrhizal fungi must be ensured through the management of site conditions conducive to the survival of fungi and maintenance of the mycorrhizal relationship (Ramsay and Dixon 2003). Soil aeration via tilling may encourage persistence of the mycorrhizal fungi since the soil at the reintroduction site has become compacted in the last 100 years (C. Knight pers. comm. 2006), probably the result of agricultural practices. The addition of fungus 
to habitat soil is the most effective way of providing sufficient mycorrhizal inocolum support in orchid translocations (Ramsay and Dixon 2003). Orchidaceous mycorrhizal fungi are not known to readily produce fruiting bodies (Rasmussen 2002) and the hyphae are too fine to observe in the rhizosphere. Therefore, the persistence of orchid mycorrhizal fungi after reintroduction to new sites has not previously been investigated. The loss of associated fungi after reintroductions of symbiotic $D$. fragrantissima, or the inability of asymbiotically germinated plants to form mycorrhizal associations after reintroduction in situ, may be one reason for the lack of survival of previously reintroduced plants.

This study investigated the effects of soil aeration and inoculation with mycorrhizal fungus on reintroduced plant survival and persistence, when planted at different times (spring, summer, autumn) and at different stages of the plant life cycle. The persistence of the mycorrhizal relationship one year after reintroduction was investigated to assess the potential longterm success of this reintroduction. Results of this research were expected to aid establishment of a reintroduced population and thereby fulfil one of the major goals of the Diuris fragrantissima recovery plan (Murphy et al. 2006).

\section{Methods}

Experimental reintroductions, involving source ex situ Diuris fragrantissima, compared planting as actively growing symbiotic seedlings in spring and autumn and as dormant tubers in summer. The spring and autumn reintroduced plants were incorporated into randomised treatments involving soil aeration and addition of support inoculum, grown on sterile millet seed. Treatment combinations were also incorporated into the experimental design. Insufficient numbers of dormant tubers were available for incorporation into all trials in the experimental reintroduction. Rather, a combination of soil aeration and support inoculum was applied to all reintroduced dormant tubers. Reintroductions were aimed for plants at 2-3 years of age, when tubers were considered to contain sufficient food storage for survival from nursery to field conditions. Site selection was based on soil properties, the previous range of $D$. fragrantissima, and conservation status, security and management of the land.

Source ex situ plants were found to be associated with fungi that were genetically similar to Tulasnella calospora and to fungi isolated from remnant in situ plants, and had the ability to initiate germination of host plants (Smith 2006). The most active fungal isolate obtained from ex situ plants, in terms of seed germination initiation, was used as the source inoculum for this research. Sterile millet seed was inoculated with this isolate for transfer to soil. Control germination trials were conducted prior to undertaking the reintroduction to ensure the viability of the fungi. All potting mix was removed from the plants prior to incorporation into $1 \mathrm{~m}^{2}$ treatment plots, five to each plot. All tuber weights were recorded prior to planting. Monthly monitoring of leaf length, width, stem height, flower and bud number and plant health was conducted between planting (September 2004, December 2004, April 2005) and April 2006.

Analysis of variance (ANOVA) was conducted on the resultant aggregated dataset using the General Linear Model: Univariate option in SPSS 14.0 (SPSS inc. 2005). Graphs were plotted using Microsoft Excel. It was hypothesised that tuber weight would be positively correlated with all variables, so where the correlation was significant, tuber weight was incorporated into the analysis as a covariate to account for any influence on results. Correlation between plants that flowered in 2004 and 2005, and those that re-emerged in seasons following flowering were examined using cross tabulations and bivariate correlations. Significance was noted when $\mathrm{p}<0.05$ for all analyses. One year after reintroduction, fungi were isolated from 20 randomly collected plants, cultured and genetically identified by direct sequencing of the ITS region. Ten of the sampled plants were reintroduced with additional inoculum and ten were reintroduced with only the fungi present in the roots at the time of planting. Seed baiting was also conducted (following Rasmussen and Whigham 1993) to determine whether fungi persisted in soil one year after reintroduction in association with host orchids and alone.

\section{Results and Discussion}

Planting as actively growing seedlings in spring and autumn was far more successful than as dor- 
mant tubers in summer, with $69 \%$ (spring) and 46\% (autumn) plants persisting after one year, compared to only $16 \%$ of plants reintroduced as dormant tubers in summer. Tuber size was positively correlated with plant size and health, confirming anecdotal reports that plants with larger tubers were more successful in reintroductions. A combination of support inoculum and soil aeration significantly improved plant growth and survival in the spring reintroduction but had no significant effect on plants reintroduced in autumn. Fungal inoculum support alone did not improve plant growth or survival in either planting time. Source plants for reintroduction were propagated asymbiotically in vitro and became associated with fungi in potting media. The fungi present in plant roots at the time of reintroduction were sufficient to support the transition from nursery to field. Therefore, the persistence of actively growing symbiotic seedlings in reintroductions shows that Diuris fragrantissima can be inoculated post-germination for successful reintroduction. Optimum monitoring dates for reintroduced $D$. fragrantissima were determined to be July, when most plants were emergent, and late October to early November, when stem height and flowering were at their greatest.

Fungi associated with Diuris fragrantissima plants at the time of reintroduction were re-isolated and identified one year after planting. Nine of ten sampled plants that were reintroduced into plots inoculated with fungi originally grown on sterile millet were found to have become associated with the support inoculum. Therefore, mycorrhizal association of symbiotic reintroduced plants was maintained for at least a year, and plants were able to form new associations with fungi post-planting. Although reintroduced plants became associated with fungi inoculated into recipient site soil, the support inoculum did not appear to increase plant growth or survival, unless combined with soil aeration for plants reintroduced in spring. The fungi present in the underground organs of $D$. fragrantissima was sufficient to support growth and flowering in two seasons.

This study was successful in the development of a reintroduction program for Diuris fragrantissima, and highlights the information necessary to implement reintroductions as effective conservation strategies. Extended monitoring of the reintroduced population is required to determine the long term success, including optimising requirements for recruitment of seedlings in situ, by means of pollination and seed germination.

ACKNOWLEDGEMENTS. The authors wish to thank the Diuris fragrantissima Recovery Team, in particular Karen Lester and Andrew Pritchard (Department of Sustainability and Environment), Colin Knight (Melbourne Zoo) and Dick Thomson (Australian Orchid Foundation) and all volunteers involved in the reintroduction. The Australian Orchid Foundation is sincerely thanked for funding $\mathrm{Z}$. Smith to attend at the IOCCIII.

\section{LITERATURE CITED}

Batty A.L., K.W. Dixon, M.C Brundrett \& K. Sivasithamparam. 2001. Long-term storage of mycorrhizal fungi and seed as a tool for the conservation of endangered Western Australian terrestrial orchids. Aust. J. Bot. 49, 619-628.

Knight, C., E.A. James, S. Akiyama \& L. Pryde. 2003. Molecular biology and pollination as a tool to enhance the long-term viability of the Sunshine Diuris (Diuris fragrantissima D. L. Jones et M. A. Clem). Final report to the Hermon Slade Foundation.

Murphy A.H., C. Knight, G. Backhouse \& A. Webster. 2006. Recovery plan for the Sunshine Diuris, Diuris fragrantissima (Orchidaceae: Diuridinae) 2002-2007. Department of Sustainability and Environment, Melbourne, Victoria, Australia.

Ramsay M.M., \& K.W. Dixon. 2003. Propagation science, recovery and translocation of terrestrial orchids. Pp. 259-288 in Dixon, K.W., S.P. Kell, R.L. Barrett, R.L. \& P.J. Cribb (eds.). Orchid conservation. Natural History Publications (Borneo): Kota Kinabalu, Sabah

Rasmussen H.N. 2002. Recent developments in the study of orchid mycorrhiza. Plant and Soil 244, 149-163.

Rasmussen H.N. \& D.F. Whigham. 1993. Seed ecology of dust seeds in situ: A new study technique and its application in terrestrial orchids. Am. J. Bot. 80, 1374-1378.

Smith, Z.F. 2006. Developing a reintroduction program for the threatened terrestrial orchid Diuris fragrantissima. Ph.D. thesis, The University of Melbourne, Victoria, Australia. 
Smith, Z.F., E.A. James \& C.B. McLean (In press). Investigation of the phylogenetic relationships between Diuris fragrantissima and its closest relatives using AFLPs. The Environmentalist.

SPSS Inc. 2005. SPSS version 14.0 for Windows. (C) SPSS Inc. Headquarters, Chicago, USA.
Vallee L., T. Hogbin, L. Monks, B. Makinson, M. Matthes \& M. Rossetto. 2004. Guidelines for the translocation of threatened plants in Australia. Australian Network for Plant Conservation: Canberra, Australia.

Zoë Smith recently obtained her Ph.D. in resource management at the University of Melbourne, Australia. Her research focussed on the taxonomic status, genetic diversity, fungal ecology and systematic relationships, and reintroduction of Diuris fragrantissima. During her studies she was employed as an orchid researcher at the Royal Botanic Gardens Melbourne, and also held a short internship at the Royal Botanic Gardens, Kew, UK, where she conducted research on species extinction modelling. Zoë is currently employed as an environmental consultant and is looking for a post doctoral position. 